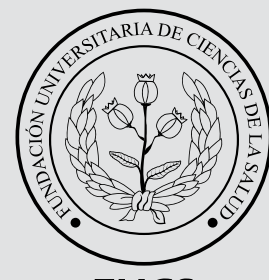

FUCS
Re

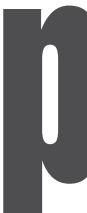

\title{
Dinámica y relación del contagio del COVID-19 después de iniciado el plan de vacunación contra el SARS-COV-2 en Colombia
}

\section{Dynamics and relationship of COVID-19 infection after SARS-COV-2 vaccination plan rollout in Colombia}

${ }^{a}$ Ingeniero. Magister en Gestión de la Tecnología Educativa, Especialista en Administración de la Informática Educativa. Docente de matemáticas e Investigador, Secretaría de Educación de Soacha, Cundinamarca.

\section{R E S U M E N}

Introducción: desde la identificación de los primeros casos de enfermedad por coronavirus 2019 (COVID-19) originada por el síndrome respiratorio agudo severo coronavirus 2 (SARS-CoV-2) en diciembre 2019, la pandemia resultante ha dado lugar a más de 130 millones de casos confirmados y más de 2,8 millones de muertes en todo el mundo a 6 de abril 2021. Objetivo: mostrar si hay alguna correlación de los contagios por COVID-19 y la vacunación contra el SARS-COV-2, entre el 17 de febrero al 17 de mayo de 2021. Metodología: la información se obtuvo de la página web del Ministerio de Salud y Protección Social, de los informes diarios de contagio y vacunación contra el COVID-19 entre el periodo comprendido entre el periodo mencionado. Resultados: el p-valor de las pruebas para las variables contagio y vacunación para COVID-19 es 0,000, menor de $\alpha=0.01$, de esta manera se acepta Ha, es decir la prueba de correlación de Pearson nos indica que existe relación entre el número de contagios por COVID-19 y la vacunación contra el SARS-COV-2 en Colombia. Conclusión: a partir de la vacunación contra COVID-19 a mediados de febrero de 2021 en Colombia, no se ha evidenciado un registro de la disminución del número de contagios por COVID-19. Este estudio aporta los primeros datos del país sobre el impacto de la vacuna contra el SARSCOV-2 en la población.

Palabras clave: correlación, Pearson, COVID-19, SARS-CoV-2, pandemia, pruebas, vacunas.

Historia del artículo:

Fecha recibido: mayo 19 de 2021 Fecha aceptado: julio 1 de 2021
Autor para correspondencia:

Ing. Jorge Enrique Díaz Pinzón jediazp@unal.edu.co
DOI

10.31260/RepertMedCir.01217372.1227 
Introduction: since the first cases of coronavirus disease 2019 (COVID-19) caused by acute respiratory syndrome coronavirus 2 (SARS-CoV-2) were identified in December 2019, the resulting pandemic has account for more than 130 million confirmed cases and more than 2.8 million deaths worldwide, as of April 6 2021. Objective: to show if there is a correlation between COVID-19 infections and SARS-COV-2 vaccination from February 17 to May 17 2021. Methodology: the information was obtained from the Ministry of Health and Social Protection website, based on COVID 19 infection and SARS-COV-2 vaccination daily reports for said period. Results: the p-value for the tests for COVID-19 infection and vaccination variables is 0.000 , which is less than $\alpha=0.01$, thus, the hypothesis ( $\mathrm{Ha}$ ) is accepted, which means that the Pearson correlation test indicates that there is a relationship between the number of COVID-19 infections and vaccination against SARS-COV-2 in Colombia. Conclusion: since vaccination against COVID-19 was started in mid-February 2021 in Colombia, there has been no evidence of a decrease in the number of COVID-19 infections. This study provides the first data in the country on how the SARS-COV-2 vaccine impacts the population.

Key words: correlation, Pearson, COVID-19, SARS-CoV-2, pandemic, tests, vaccines.

(C) 2021 Fundación Universitaria de Ciencias de la Salud - FUCS. This is an open access article under the CC BY-NC-ND license (http://creativecommons.org/licenses/by-nc-nd/4.0/).

\section{INTRODUCCIÓN}

Desde la identificación de los primeros casos de enfermedad por coronavirus 2019 (COVID-19) originada por el síndrome respiratorio agudo severo coronavirus 2 (SARS-CoV-2) en diciembre de 2019, la pandemia resultante ha dado lugar a más de 130 millones de casos confirmados de COVID-19 y más de 2,8 millones de muertes en todo el mundo al 6 de abril de 2021. ${ }^{1-3}$

El desarrollo de una cartera de vacunas COVID-19 para inmunizar a la población mundial sigue siendo un imperativo urgente de salud pública. ${ }^{4}$ Se necesita con urgencia una campaña de vacunación eficaz y segura para detener la rápida propagación del síndrome respiratorio agudo severo coronavirus 2 (SARS-CoV-2) y la enfermedad resultante, COVID-19.5

Desde que se secuenció genoma del coronavirus 2 del síndrome respiratorio agudo severo (SARS-CoV-2), los investigadores se apresuraron a desarrollar vacunas para frenar la propagación del COVID-19.6,7 Dada la inviabilidad de los bloqueos a largo plazo8,9 y el rastreo de contactos efectivo en un gran número de casos así como la disponibilidad de varias vacunas COVID-19 aprobadas, muchos países han invertido en aplicaciones masivas.

Para conseguir una reducción sostenida de los casos de infección ${ }^{10}$ se necesitan contramedidas múltiples, incluido el distanciamiento, las pruebas y el rastreo, más aún a la luz de la reciente aparición de nuevas variantes del SARSCoV-2 ${ }^{11}$, como B.1.1.7 y B.1.351, que según se informa tienen una mayor transmisibilidad ${ }^{12,13}$ y posiblemente causan una enfermedad más grave ${ }^{14}$ en comparación con la cepa original. No se espera que la vacunación por sí sola pueda controlar la propagación de la infección, por lo tanto es necesario coordinar una campaña de vacunación cuidadosamente planificada. ${ }^{15,16} \mathrm{Al}$ cumplirse tres meses de la campaña de vacunación en Colombia, se han aplicado cerca de 7'537.763 dosis acumuladas y 2' 885.758 segundas dosis contra el nuevo coronavirus, lo que equivale al 9,12\% de la población.

\section{Correlación lineal de Pearson}

La cuantificación de la fuerza de la relación lineal entre dos variables cuantitativas se estudia por medio del cálculo del coeficiente de correlación de Pearson. Este oscila entre - 1 y +1 . Un valor de -1 indica una relación lineal o línea recta positiva perfecta. Una correlación próxima a cero indica que no hay relación lineal entre las dos variables. ${ }^{12}$ En la tabla 1 se aprecia la escala de coeficiente de correlación de Pearson. ${ }^{17}$

Tabla 1. Escala de coeficiente de correlación de Pearson

\begin{tabular}{|c|l|}
\hline Valor & \multicolumn{1}{|c|}{ Significado } \\
\hline-1 & Correlación negativa grande y perfecta \\
$-0,9$ a $-0,99$ & Correlación negativa muy alta \\
$-0,7$ a $-0,89$ & Correlación negativa alta \\
$-0,4$ a $-0,69$ & Correlación negativa moderada \\
$-0,2$ a $-0,39$ & Correlación negativa baja \\
$-0,01$ a $-0,19$ & Correlación negativa muy baja \\
0 & Correlación nula \\
0,01 a 0,19 & Correlación positiva muy baja \\
0,2 a 0,39 & Correlación positiva baja \\
0,4 a 0,69 & Correlación positiva moderada \\
0,7 a 0,89 & Correlación positiva alta \\
0,9 a 0,99 & Correlación positiva muy alta \\
1 & Correlación positiva grande y perfecta \\
\hline Fuente: Suárez
\end{tabular}

El objetivo de esta investigación es mostrar si hay alguna correlación de los contagios por COVID-19 y la vacunación contra el SARS-COV-2 entre el periodo comprendido entre el 17 de febrero al 17 de mayo de 2021. 


\section{METODOLOGÍA}

El trabajo de investigación se desarrolló mediante un tipo experimental que es el siguiente: "aquella que permite mayor seguridad al establecer relaciones de causa a efecto pues presenta una visión general y aproximada del objeto de estudio, además de contar con una investigación cuyo diseño establece un método experimental habitual del conjunto de las normas científicas", Monje (2011) citado por Díaz. ${ }^{18}$ Según Shuttleworth citado por Díaz, ${ }^{19,20}$ menciona que "regularmente a estos experimentos se los nombra ciencia verdadera y manejan medios matemáticos y estadísticos cotidianos para evaluar los resultados de modo concluyente. Todos los experimentos cuantitativos utilizan un formato estándar con algunas pequeñas diferencias interdisciplinarias para generar una hipótesis que será probada o desmentida. Esta hipótesis debe ser demostrable por medios matemáticos y estadísticos y constituye la base alrededor de la cual se diseña todo el experimento".

\section{POBLACIÓN}

La información se obtuvo de la página web del Ministerio de Salud y Protección Social ${ }^{21}$ de los informes diarios de vacunación contra el COVID-19, en el periodo mencionado.

\section{FORMULACIÓN DE HIPÓTESIS}

La hipótesis del presente trabajo de investigación se diseña como una relación causal y se enuncia de la siguiente forma: (a) hipótesis alterna ( $\mathrm{Ha})$, existe relación entre el número de contagios por COVID-19 y la vacunación contra el SARS-COV-2 en Colombia. (b) hipótesis nula (Ho), no existe relación entre el número de contagios por COVID-19 y la vacunación contra el SARS-COV-2 en Colombia. Nivel de significancia: $=1 \%=0,1$. Toma de decisión: sí el p- valor $<$ a es aceptar Ha, entonces rechazamos la hipótesis nula y nos quedamos con la hipótesis del investigador (Ha).

\section{RESULTADOS}

En la figura 1 se aprecian los casos diarios de contagio SARS-2 y la vacunación contra COVID-19 durante el período del 17 de febrero 2021 hasta el 17 de mayo 2021. Se describe en ella una semejanza en la tendencia de las líneas de contagio (azul) y la vacunación (naranja). Es decir, mientras aumenta el número de contagios en el tiempo también aumenta el número de vacunas.

En la tabla 2 se aprecia el p-valor de las pruebas para las variables muestras de contagio y vacunación contra COVID-19 que es 0,000, menor de $\alpha=0,1$, de esta manera

250000

25000

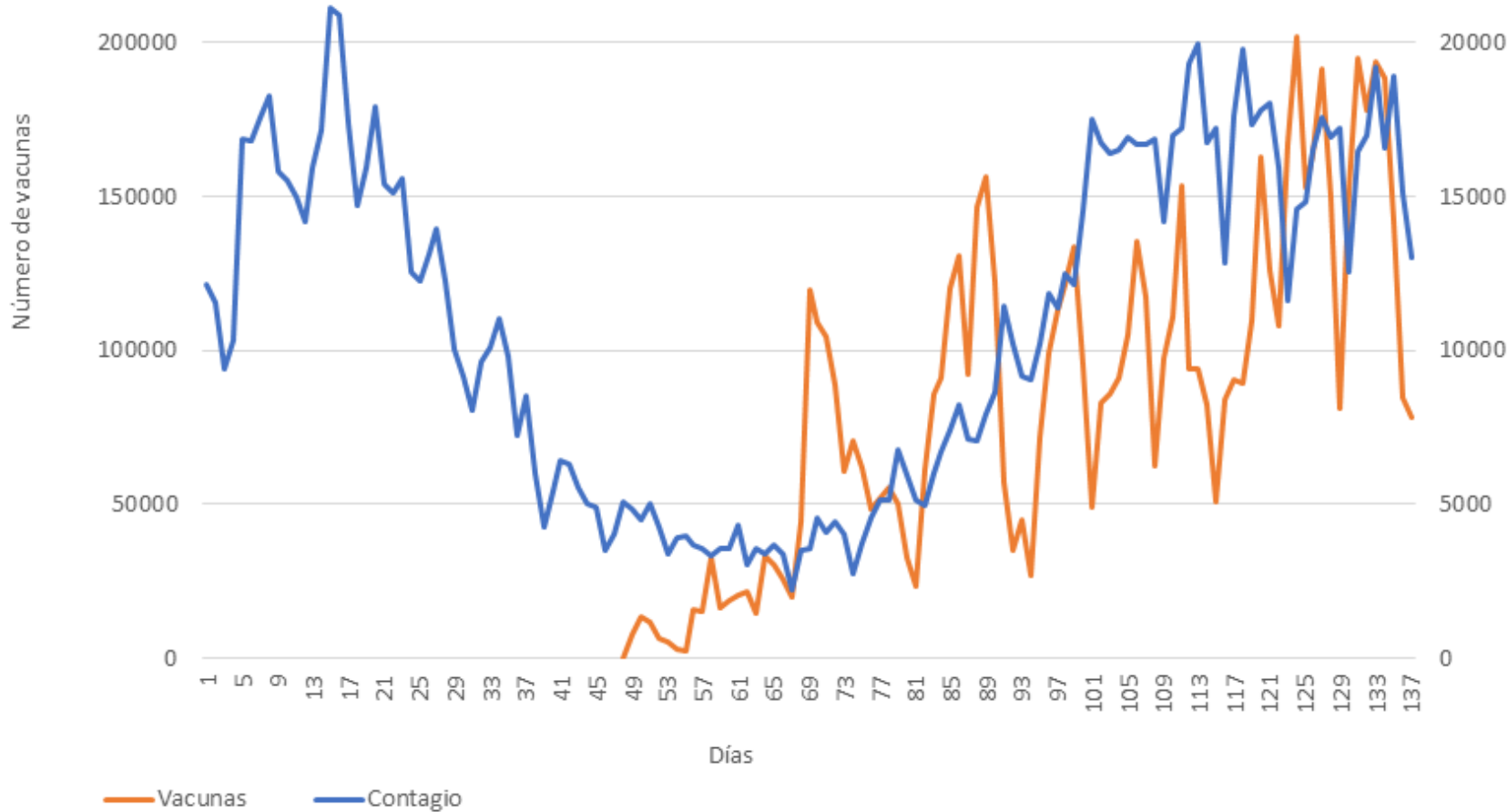

Figura 1. Vacunación y contagio. Fuente: el autor. 
se acepta Ha, es decir la prueba de correlación de Pearson nos indica que existe relación entre el número de contagios por COVID-19 y la vacunación contra el SARS-COV-2 en Colombia durante el período mencionado.

En la figura 2 se aprecia el diagrama de correlación entre contagio por COVID-19 y la vacunación contra SARSCOV-2. El número de datos es igual a 90; $\mathrm{r}=0,651 ; \mathrm{p}<$ 0,001 . De esta manera según el valor $r=0,651$, se evidencia una correlación positiva moderada entre las variables. La ecuación lineal está dada por: $\mathrm{Y}=6,01 \mathrm{X}+2,17 \mathrm{E} 4$ lo que se traduce que en la medida que aumentan los valores de contagio por COVID-19, también se eleven los casos de vacunación por SARS-COV-2, y viceversa.
Tabla 2. Correlación de Pearson

\begin{tabular}{|l|l|c|c|}
\hline \multirow{4}{*}{ Vacunación-día } & Correlación de Pearson & 1 & Contagio \\
& Sig. (bilateral) & &, $651^{\star \star}$ \\
& $\mathrm{N}$ & 90 &, 000 \\
\cline { 1 - 1 } Contagio & Correlación de Pearson &, $651^{\star \star}$ & 90 \\
& Sig. (bilateral) &, 000 & 1 \\
& $\mathrm{~N}$ & 90 & 90 \\
\hline
\end{tabular}

**La correlación es significativa en el nivel 0,01 (bilateral). Fuente. el autor.

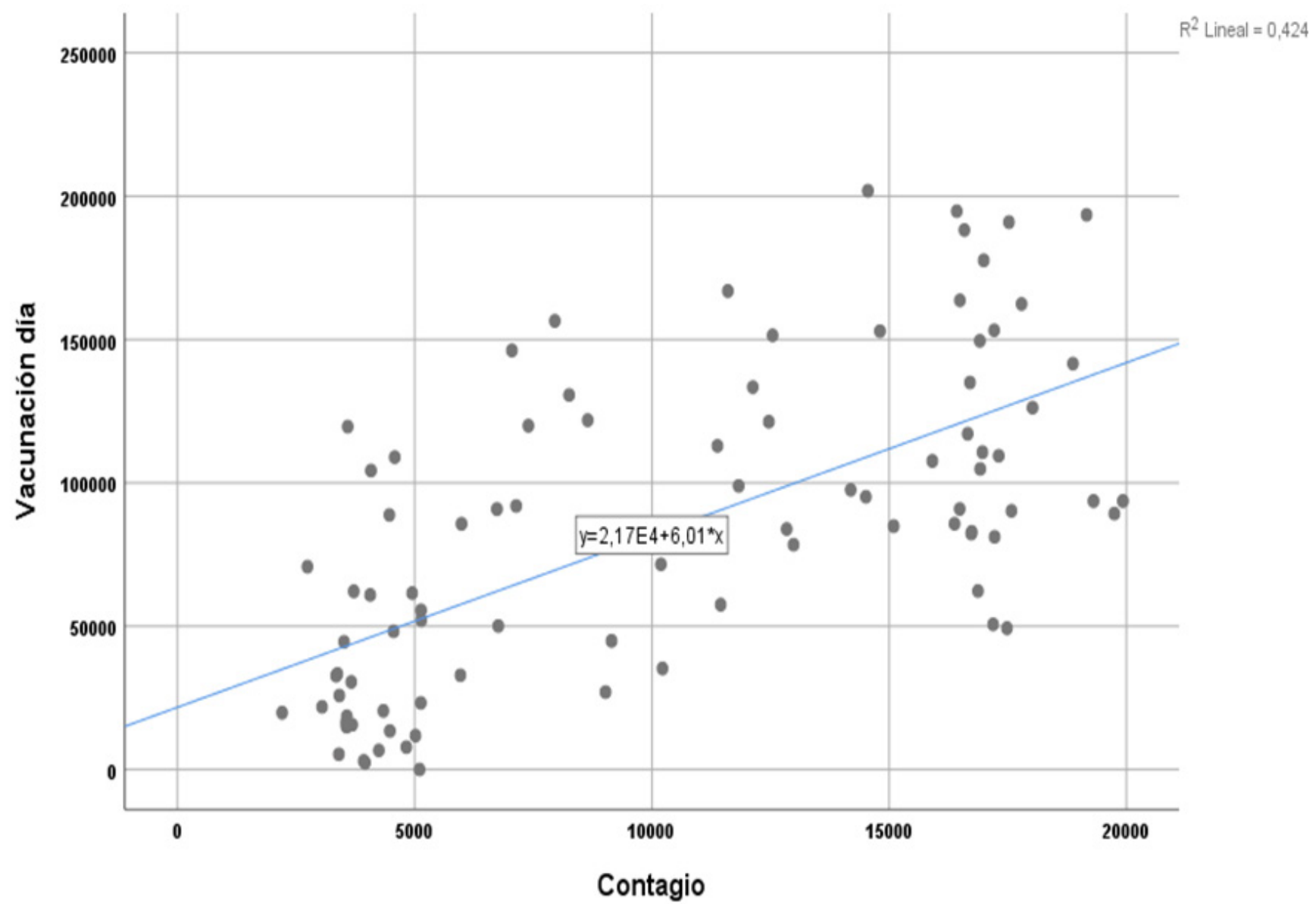

Figura 2. Diagrama de correlación por contagio y vacunación. Fuente: el autor

\section{CONCLUSIONES}

A partir de los datos analizados se concluye que el contagio por COVID-19 se relaciona con la vacunación contra SARSCOV-2, es decir, que en la medida que aumentan los valores de vacunación, también se incrementan los casos de contagio por COVID-19 y viceversa.
Los resultados obtenidos en este trabajo de investigación descubren que se ha presentado un aumento en los nuevos contagios por SARS-COV-2 registrados en Colombia durante el período posterior al inicio de la campaña de vacunación, en comparación con los meses de enero y febrero 2021. Esto indica un alza significativa a la observada en la población general, sugiriendo que no ha ocurrido hasta el momento un efecto de la inmunización en la dinámica de contagios 
por COVID-19. Este estudio aporta los primeros datos del país sobre el impacto de la vacuna contra el SARS-COV-2 en la población, que demuestran como la campaña aún no ha sido efectiva. Aunque el hallazgo es preliminar, tiene importante implicación en la salud pública para el combate contra la pandemia de COVID-19. Se requieren más estudios predestinados a evaluar la eficacia de la vacunación en la reducción de la transmisión del SARS-CoV-2, tanto a nivel individual como a nivel poblacional, con un mayor rastreo longitudinal y en poblaciones adicionales.

\section{REFERENCIAS}

1. Gudbjartsson DF, Helgason A, Jonsson H, Magnusson OT, Melsted P, Norddahl GL. Spread of SARS-CoV-2 in the Icelandic Population. N Engl J Med. 2020;382(24):2302-2315. doi: 10.1056/ NEJMoa2006100.

2. Huang C, Wang Y, Li X, Ren L, Zhao J, Hu Y, Zhang L, et al. Clinical features of patients infected with 2019 novel coronavirus in Wuhan, China. Lancet. 2020;395(10223):497-506. doi: 10.1016/ S0140-6736(20)30183-5

3. World Helath Organization. WHO Coronavirus (COVID-19) Dashboard [Internet]. World Helath Organization; 2021 [consultado 6 de abril 2021]; Disponible en: https://covid19.who. int/.

4. Krammer F. SARS-CoV-2 vaccines in development. Nature. 2020;586(7830):516-527. doi: 10.1038/s41586-020-2798-3

5. Walsh EE, Frenck Jr RW, Falsey AR, Kitchin N, Absalon J, Gurtman A, et al. N Engl J Med. 2020;383(25):2439-2450. doi: 10.1056/NEJMoa202790

6. Wang J, Peng Y, Xu H, Cui Z, Williams RO. The COVID-19 Vaccine Race: Challenges and Opportunities in Vaccine Formulation. AAPS PharmSciTech. 2020;21(6):225. doi: 10.1208/s12249-020-01744-7

7. Rawat K, Kumari P, Saha, L. COVID-19 vaccine: A recent update in pipeline vaccines, their design and development strategies. Eur J Pharmacol. 2021;892:173751. doi: 10.1016/j.ejphar.2020.173751

8. Abbasi K. Behavioural fatigue: a flawed idea central to a flawed pandemic response. BMJ. 2020;370:m3093. doi: 10.1136/bmj. m3093

9. Rypdal K, Bianchi FM, Rypdal, M. Intervention Fatigue is the Primary Cause of Strong Secondary Waves in the COVID-19 Pandemic. Int J Environ Res Public Health. 2020;17(24):9592. doi: 10.3390/ijerph17249592
10. Priesemann V, Brinkmann MM, Ciesek S, Cuschieri S, Czypionka T, et al. Calling for pan-European commitment for rapid and sustained reduction in SARS-CoV-2 infections. Lancet. 2021;397(10269):9293. doi: 10.1016/S0140-6736(20)32625-8

11. Priesemann V, Brinkmann MM, Ciesek S, Cuschieri S, Czypionka T, et al. Calling for pan-European commitment for rapid and sustained reduction in SARS-CoV-2 infections. Lancet. 2021;397(10269):9293. doi: 10.1016/S0140-6736(20)32625-8

12. Davies NG, Abbott S, Barnard RC, Jarvis CI, Kucharski AJ, et al. Estimated transmissibility and impact of SARS-CoV-2 lineage B.1.1.7 in England. Science. 2021;372(6538):eabg3055. doi: 10.1126/science.abg 3055

13. Abbott S, Funk S, CMMID COVID-19 Working Group. Local area reproduction numbers and S-gene target failure [Internet]. Centre for Mathematical Modelling of Infectious Diseases 2021. [Citado 10 de junio de 2021]; Disponible en: https://cmmid.github.io/ topics/covid19/local-r-sgtf.html

14. Horby P, Huntley C, Davies N, Edmunds J, Ferguson N, Medley G, Semple C. NERVTAG paper on COVID-19 variant of concern B.1.1.7. Department of Health and Social Care. [Citado 10 de junio de 2021]; Disponible en: https://www.gov.uk/government/ publications/nervtag-paper-on-covid-19-variant-of-concern-b117

15. Bubar KM, Reinholt K, Kissler SM, Lipsitch M, Cobey S, Grad YH. Model-informed COVID-19 vaccine prioritization strategies by age and serostatus. Science. 2021;371(6532):916-921. doi: 10.1126/ science.abe6959

16. Ramos AM, Vela-Pérez M, Ferrández MR, Kubik AB, Ivorra B. Modeling the impact of SARS-CoV-2 variants and vaccines on the spread of COVID-19. Preprint in ResearchGate. [Citado 10 de junio de 2021]; Disponible en: https://doi.org/10.13140/ RG.2.2.32580.24967/2

17. Suárez-Ibujes M. Coeficiente de correlación de Karl Pearson [Internet]. 2021. [citado 14 mayo 2021]; Disponible en: https:// www.monografias.com/trabajos85/coeficiente-correlacion-karlpearson/coeficiente-correlacion-karl-pearson.shtml

18. Díaz Pinzón, JE. Medidas de frecuencia por COVID-19 en Bogotá DC. Repert Med Cir. 2020;29(Núm. Supl.1):94-98. https://doi. org/10.31260/RepertMedCir.01217372.1110

19. Díaz Pinzón JE. Estimación de la prevalencia del COVID-19 en Colombia. Repert Med Cir. 2020;29(Núm. Supl.1):99-102. https:// doi.org/10.31260/RepertMedCir.01217372.1115

20. Díaz Pinzón JE. Estimación de las tasas de mortalidad y letalidad por COVID-19 en Colombia. Repert Med Cir. 2020;29(Núm. Supl.1):8993. https://doi.org/10.31260/RepertMedCir.01217372.1103 